\title{
Precisão da estimativa da massa de forragem com discos medidores em pastagem nativa
}

\author{
Accurate estimate of forage mass with discs meter in native pasture
}

\section{Caius Barcellos de Pellegrini' ${ }^{\mathrm{I}}$ Eduardo Londero Moojen ${ }^{\mathrm{I}{ }^{*}}$ José Henrique Souza da Silva $^{\mathrm{I}}$ Marta Gomes da Rocha ${ }^{\mathrm{I}}$ Marcos da Silva Brum ${ }^{\mathrm{I}}$ Fernando Segala Gravina ${ }^{\mathrm{I}}$}

RESUMO

Este trabalho foi realizado de outubro a abril de 2005 a fim de avaliar e comparar a precisão da estimativa da massa de forragem (MF) com o emprego de discos medidores de forragem, com diferentes áreas e pesos em pastagem nativa $(P N)$. Os tratamentos foram três diferentes áreas de disco: 0,1; 0,2; e 0,3m², cada uma combinada com três pesos de disco: 5, 10 e $15 \mathrm{~kg} \mathrm{~m}^{-2}$. O delineamento experimental foi em blocos casualizados, em esquema de parcelas subdivididas. Os resultados obtidos foram submetidos à análise de regressão entre medidas do disco (área e peso de disco), e a MF foi determinada em cada período de avaliação. Do modelo matemático obteve-se o coeficiente de variação residual $(\mathrm{CV})$. A determinação do procedimento de avaliação de modelos de regressão para os CVs obtidos, em relação às áreas e aos pesos de disco, foi definida pelo coeficiente de determinação e pela variância residual. As relações entre as combinações das três áreas dos discos associadas com os três pesos e o CV das medidas obtidas com disco foram quadráticas positivas. As relações entre os pesos adicionados sobre as áreas dos discos e o CV foram lineares positivas. $O$ avanço dos períodos avaliados aumentou linearmente o CV na quantificação da $M F$ da $P N$ com disco medidor. $O$ disco de menor área $\left(0,1 \mathrm{~m}^{2}\right)$ e com peso de $5 \mathrm{~kg} \mathrm{~m}^{-2}$ apresentou o menor $\mathrm{CV}$, sendo, portanto, o mais indicado para avaliar a MF da PN.

Palavras-chave: área de disco medidor, coeficiente de variação residual, espécies nativas, pastagens, peso de disco medidor.

\section{ABSTRACT}

This research was conducted between October and April of 2005, with the objective of evaluating and comparing the efficiency forage mass estimate (FM) with different areas and weights of the discs meter in native pasture (NP). The treatments were three different discs areas, $0.1,0.2$ and $0.3 \mathrm{~m}^{2}$, each one combined with three weights of discs 5,10 and $15 \mathrm{~kg}$ $\mathrm{m}^{-2}$. The experimental design was block randomized in a splitplot. The obtained results were submitted to regression analysis between meter of disc (area and weight disc) and FM determined in each period of evaluation. From the mathematical model it was obtained the coefficient of residual variation (CV). The regression models were determined by coefficient of determination and residual variance. The relationship between three areas of discs associated with three weights and the CV of the measures obtained with discs were quadratic positive. The relations between the three weights and areas of discs meter and the $C V$ were linear positive. The advance of the evaluation period increased linear the CV for determining FM in NP. The disc of smaller area, $0.1 \mathrm{~m}^{2}$ and weight of $5 \mathrm{~kg} \mathrm{~m}^{-2}$, presented smaller $C V$ for MF estimate in NP, therefore the most indicated to predict of $M F$ in NP.

Key words: disc area, coefficient of residual variation, native species, grasslands, disc weight.

\section{INTRODUÇÃO}

A pastagem nativa cobre uma área aproximada de 10,5 milhões de hectares (IBGE, 2006) e constitui o grande recurso forrageiro responsável pela produção bovina e ovina no Rio Grande do Sul (RS). O manejo correto e eficiente dessa pastagem passa pelo controle do ajuste da oferta de forragem, que representa adequação de uma disponibilidade de forragem a uma carga animal (CAUDURO et al., 2006).

Para o cálculo da oferta de forragem, a massa de forragem (MF) deve ser medida. De acordo com FRAME (1981), em experimentos envolvendo

IUniversidade Federal de Santa Maria, (UFSM), Santa Maria, RS, Brasil.

IIDepartamento de Zootecnia, UFSM. Av. Roraima, 1000, 97105-900, Santa Maria, RS, Brasil. E-mail:moojenel@smail.ufsm.br. Autor para correspondência. 
produção animal em pastagens, a falta de medições de MF significa que informações importantes sobre os efeitos do manejo sobre o acúmulo, a taxa de acúmulo ou o consumo simplesmente não são geradas, o que compromete enormemente a interpretação dos resultados. 'T MANNETJE (2000) ressaltou a importância do monitoramento da variação da MF como uma das formas mais efetivas de gerar subsídios para diversos processos de gerenciamento e tomada de decisão sobre o manejo do pastejo.

O método do corte é o que apresenta maior precisão da estimativa da MF, pois as amostras de área delimitada são cortadas rente ao solo, armazenadas e secas para a determinação da matéria seca (MS). No entanto, o corte de amostras demanda trabalho, que limita o número de amostras nas avaliações e diminui a precisão. Por essas limitações, métodos indiretos de amostragem foram desenvolvidos. Dentre os métodos indiretos de predição de MF, está o disco medidor de forragem.

O disco medidor consiste em um componente (quadrado ou redondo) de metal de área e peso conhecidos, o qual desliza ao longo de um eixo central. A altura da forragem acima do solo é mensurada pelo eixo central, que possui uma escala graduada, sendo medida por um contador. Esse instrumento avalia a MF considerando sua altura e densidade em função da pressão exercida pelo disco medidor sobre a pastagem. Segundo 'T MANNETJE (2000), a combinação de duas características da pastagem, como altura e densidade, incrementam a precisão da estimativa de forragem, sendo mais vantajoso que a utilização de apenas uma característica, como a altura.

Em grandes extensões de terra, o fácil manuseio do disco medidor de forragem possibilita leituras rápidas, com muitas amostragens. Para PEDREIRA (2002), o disco medidor de forragem é, provavelmente, uma técnica indireta mais eficiente para medir MF de dosséis de porte médio a baixo, de espécies folhosas e de colmos macios. Conforme o mesmo autor, em dosséis com colmos muito grandes e rígidos, a leitura pode não levar em conta a densidade, mas responder apenas à altura, resultando em correlações fracas entre alturas das medidas do disco e MF da pastagem quantificada.

BARCELLOS (1990) obteve dificuldade de desenvolver equações de regressão precisas com disco medidor (área $0,25 \mathrm{~m}^{2}$; peso $9,2 \mathrm{~kg} \mathrm{~m}^{-2}$ ) em pastagem nativa submetida a diferentes métodos e pressões de pastejo durante épocas do ano. No mesmo ambiente de pesquisa, GOMES et al. (1998) relacionaram a presença de colmos rígidos das espécies cespitosas à baixa correlação das leituras obtidas com disco medidor na predição de MF da pastagem nativa.
As pesquisas desenvolvidas com o emprego de disco medidor de forragem não determinaram a área e o peso de disco ideal para estimativa da MF da pastagem nativa. Nesse sentido, o objetivo deste trabalho foi verificar a precisão da estimativa da MF, por meio do emprego de discos medidores, com diferentes áreas e pesos em pastagem nativa submetida ao pastejo de bovinos de corte.

\section{MATERIAL E MÉTODOS}

O experimento foi conduzido em área de pastagem nativa, no município de Dilermando de Aguiar, Rio Grande do Sul (RS), região fisiográfica denominada Depressão Central. As avaliações foram realizadas nas seguintes datas: 07/10/2004, 06/11/04, 04/12/04, 01/01/2005, 15/02/05, 19/03/05 e 16/04/05.

Os tratamentos foram formados pelas combinações de três diferentes áreas de disco $(0,1 ; 0,2$ e $\left.0,3 \mathrm{~m}^{2}\right)$ e três pesos de disco $\left(5,10\right.$ e $\left.15 \mathrm{~kg} \mathrm{~m}^{-2}\right)$ discriminados a seguir: D1A5 $\left(0,1 \mathrm{~m}^{2}\right.$ e $\left.5 \mathrm{~kg} \mathrm{~m}^{-2}\right), \mathrm{D} 1 \mathrm{~A} 10$ $\left(0,1 \mathrm{~m}^{2}\right.$ e $\left.10 \mathrm{~kg} \mathrm{~m}^{-2}\right)$, D1A15 $\left(0,1 \mathrm{~m}^{2}\right.$ e $\left.15 \mathrm{~kg} \mathrm{~m}^{-2}\right)$, D2A5 $\left(0,2 \mathrm{~m}^{2}\right.$ e $\left.5 \mathrm{~kg} \mathrm{~m}^{-2}\right), \mathrm{D} 2 \mathrm{~A} 10\left(0,2 \mathrm{~m}^{2}\right.$ e $\left.10 \mathrm{~kg} \mathrm{~m}^{-2}\right), \mathrm{D} 2 \mathrm{~A} 15$ $\left(0,2 \mathrm{~m}^{2}\right.$ e $\left.15 \mathrm{~kg} \mathrm{~m}^{-2}\right), \mathrm{D} 3 \mathrm{~A} 5\left(0,3 \mathrm{~m}^{2} \mathrm{e} 5 \mathrm{~kg} \mathrm{~m}^{-2}\right), \mathrm{D} 3 \mathrm{~A} 10\left(0,3 \mathrm{~m}^{2}\right.$ e $\left.10 \mathrm{~kg} \mathrm{~m}^{-2}\right)$ e D3A15 $\left(0,3 \mathrm{~m}^{2}\right.$ e $\left.15 \mathrm{~kg} \mathrm{~m}^{-2}\right)$.

A unidade experimental correspondeu a um ponto aleatório distinto, com distância média de 20 metros, aproximadamente, nas duas linhas longitudinais do piquete demarcadas no início do experimento. As áreas anteriormente avaliadas foram excluídas, bem como os dejetos de bovinos. O método de pastejo utilizado foi o contínuo, com uma taxa de lotação de $0,8 \mathrm{UA} \mathrm{ha}^{-1}$ no período experimental.

As medidas da pastagem foram obtidas descendo-se suavemente o conjunto do disco de alumínio sobre a vegetação, no momento em que se efetuava a leitura do contador do aparelho; em seguida, o eixo central metálico do equipamento era empurrado em direção ao solo. No momento em que a base metálica do eixo central entrasse em contato com o solo, esta era puxada de volta a sua posição original. Após esse procedimento, a leitura do contador era novamente efetuada. O valor mensurado do aparelho foi calculado, subtraindo-se a leitura final do contador da leitura inicial. Entre o momento da deposição do primeiro disco de área $0,1 \mathrm{~m}^{2}$ e peso $5 \mathrm{~kg} \mathrm{~m}^{-2}$ foram aguardados cerca de cinco segundos (tempo médio entre cada leitura), para que ocorresse a acomodação da vegetação e estabilização da leitura correspondente. Após a leitura dessa área de disco, sobre o disco foram adicionados pesos de 5 e $10 \mathrm{~kg} \mathrm{~m}^{-2}$, e obtidas as respectivas leituras das medidas da forragem. Posteriormente, no ponto 
inicial, foi colocado o disco de área $0,2 \mathrm{~m}^{2}$ e peso $5 \mathrm{~kg} \mathrm{~m}^{-2}$ para a tomada da leitura da área determinada. Sobre o disco de área $0,2 \mathrm{~m}^{2}$ e peso $5 \mathrm{~kg} \mathrm{~m}^{-2}$ foram acrescentados pesos de 5 e $10 \mathrm{~kg} \mathrm{~m}^{-2}$ para a tomada das leituras da forragem. As mesmas operações foram realizadas com o disco de área $0,3 \mathrm{~m}^{2}$ e peso $5 \mathrm{~kg} \mathrm{~m}^{-2}$, e acrescentados posteriormente pesos de 5 e $10 \mathrm{~kg} \mathrm{~m}^{-2}$ para a tomada das leituras sucessivas da forragem da pastagem nativa. Por ocasião das amostragens, após a coleta das medidas de altura de cada área e peso de disco, as áreas de discos foram retiradas, e as amostras das áreas de disco determinadas foram demarcadas com três aros de áreas circulares, respectivamente, 0,$1 ; 0,2$ e $0,3 \mathrm{~m}^{2}$, e cortadas com tesoura de esquilar rente ao solo. Todos os pontos de amostragem (50) foram cortados nas três áreas de disco, em cada unidade experimental do experimento, e as amostras foram armazenadas em sacos de papel e levadas à estufa com circulação forçada de ar a $65^{\circ} \mathrm{C}$, por $72 \mathrm{~h}$, para determinação da matéria seca (MS).

A amostragem das espécies que compõem a vegetação da pastagem nativa foi realizada por meio do método BOTANAL (TOTHILL et al., 1992). Esse método constitui-se da combinação de estimativas da composição relativa de espécies e do rendimento da pastagem. Tomou-se a frequência de espécies por meio do critério de presença/ausência nos quadros avaliados.

Os resultados obtidos foram submetidos à análise de regressão entre altura da pastagem (Yi) e MF (Xi) determinada em cada período de avaliação, área e peso de disco. $\mathrm{O}$ modelo estatístico adotado foi: $\hat{\mathrm{Y}} \mathrm{i}=\beta 0+\beta \mathrm{i}+\hat{\mathrm{i}} \mathrm{i}$, em que: $\hat{\mathrm{Y}} \mathrm{i}$ refere-se à altura no período, à área e ao peso de disco; $\beta 0+\beta i$ referem-se a parâmetros estimados da equação; e î i refere-se a erro experimental. Desse modelo paramétrico foram determinados os CV's por área e o peso do disco (desvio padrão residual expresso na porcentagem da média).

A seguir, foi adotado o método da análise de variância dos CV's do modelo descrito anteriormente, delineamento blocos ao acaso em esquema de parcelas subdivididas com as áreas na parcela e os pesos nas subparcelas. O modelo estatístico adotado foi: $\hat{Y} i j k=\mu$ $+ß k+a ́ i+o ̈ j+(a ́ ß) i k+(a ́ o ̈) i j+\hat{~} i j k, ~ e m ~ q u e: ~ Y i j k$ refere-se a CV's residuais da área $i$, ao peso $\mathrm{j}$ e ao período $\mathrm{k} ; \mu$ refere-se à média de todas as observações; $ß \mathrm{k}$ refere-se ao efeito do período k; ai refere-se ao efeito da área $\mathrm{i}$; öj refere-se ao efeito do peso j; (á $ß)$ ik refere ao erro (a), interação entre área i e período k; (áö) ij refere-se à interação entre área $\mathrm{i}$ e peso $\mathrm{j}$; e î ijk referese à variação aleatória residual, que serve para testar pesos e a interação área e peso.
Quando os efeitos principais desses fatores foram significativos e não houve interação entre área e peso de disco, ajustou-se uma equação de regressão polinomial até o segundo grau em função dos períodos (dias), das áreas e dos pesos. No entanto, quando a interação entre área e peso de disco foi significativa, ajustou-se uma equação polinomial até segundo grau em função dos pesos em cada área e vice-versa. $\mathrm{O}$ modelo estatístico adotado foi: $\hat{Y} i j=\beta 0+\beta 1 X i j+\beta 2$ $X i{ }^{2}+\hat{i}$ ij, em que: $\hat{Y} i j$ são os coeficientes de variação obtidos nos modelos anteriores de regressão; $\beta 0, \beta 1 \mathrm{e}$ ß2 são parâmetros estimados da equação; Xij é a área ou o peso do disco; Xij ${ }^{2}$ são desvios da regressão obtidos entre a soma de quadrados dos modelos de regressão e comparação de médias; î ij são desvios da regressão (lack of fit) separados do erro îijk, determinado no primeiro modelo. Esses dois erros constituem o resíduo do modelo de regressão.

Os dados foram submetidos à análise de variância, e as médias foram comparadas pelo teste de Tukey, no nível de 5\% de probabilidade. As análises foram efetuadas com auxílio do aplicativo estatístico SAS (2001).

\section{RESULTADOS E DISCUSSÃO}

As relações entre o coeficiente de variação residual e as combinações das três áreas dos discos com os três pesos, e entre o coeficiente de variação residual e os pesos adicionados sobre as áreas dos discos foram quadráticas positivas e lineares positivas, respectivamente (Figura 1).

Os aumentos das áreas dos discos influenciaram o CV (Figura 1a).

A área de disco de $0,1 \mathrm{~m}^{2}$ e com peso de $5 \mathrm{~kg}$ $\mathrm{m}^{-2}$ apresentou o menor $\mathrm{CV}$ das leituras obtidas com disco medidor de forragem na pastagem nativa, nos períodos avaliados. Conforme aumentou a área do disco, aumentou o CV de forma quadrática ascendente para os pesos de 10 e $15 \mathrm{~kg} \mathrm{~m}^{-2}$. A menor oscilação do $\mathrm{CV}$ foi observada no menor peso de disco $5 \mathrm{~kg} \mathrm{~m}^{-2}$ $(32,8 \% ; 36,6$ e 36,8$)$ à medida que as áreas de disco aumentaram. Para os pesos de 10 e $15 \mathrm{~kg} \mathrm{~m}^{-2}$ relacionados com as três áreas de disco, os CVs oscilaram com resultados bastantes altos, de 48,5 a $81 \%$. No entanto, observando as equações de regressão, para cada peso de disco com aumento da área, estas apresentaram valores de coeficientes de determinação $\left(\mathrm{R}^{2}\right)$ maiores que $80 \%$ para os pesos de 10 e $15 \mathrm{~kg} \mathrm{~m}^{-2}$ e $44 \%$ para peso $5 \mathrm{~kg} \mathrm{~m}^{-2}$. O aumento do $\mathrm{CV}$ verificado com o aumento das áreas dos discos pode estar associado aos atributos do pasto, à heterogeneidade espacial da vegetação, à morfologia 


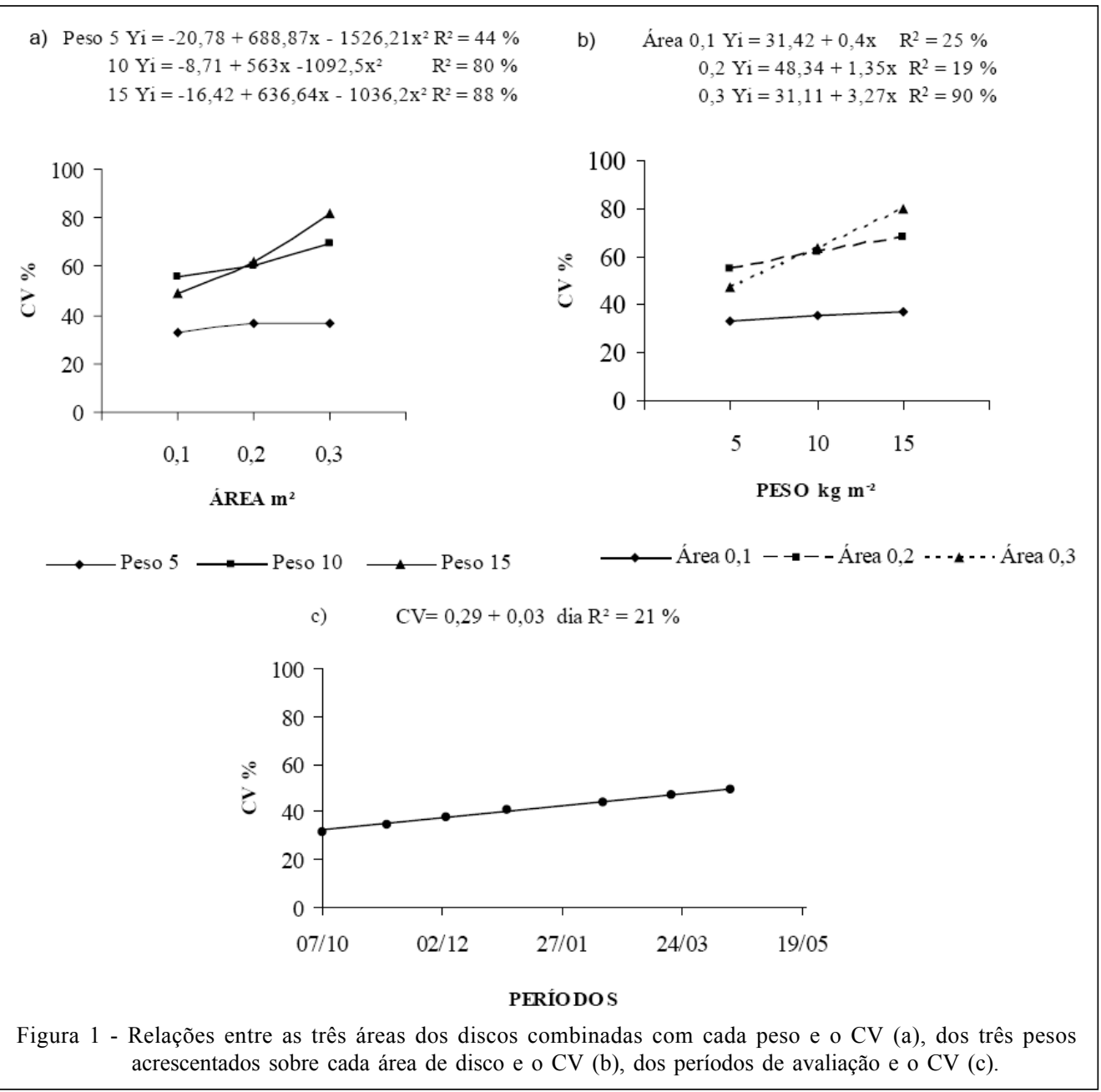

e à altura das espécies da vegetação da pastagem nativa obtidas nas leituras com disco. Outros fatores que promovem uma complexa heterogeneidade da vegetação nativa derivam das alterações microambientais e estão associados ao ciclo fenológico das espécies e ao pastejo seletivo promovido pelos herbívoros (BARCELLOS, 1990). WILM et al. (1944) relataram que a quantificação da produção e utilização da forragem, na experimentação, encontram seus pontos críticos nas características das espécies e na variabilidade da população.

A contribuição das espécies das diferentes famílias na MS da pastagem nativa aumentou a variabilidade nas medidas obtidas com disco à medida que se aumentou a área do disco. As espécies com maior participação na MS da pastagem nativa pertenciam à família Gramineae (Poaceae) $(69,2 \% \mathrm{MS})$. As espécies das famílias Leguminosae (Fabaceae), Compositae (Asteraceae) e outras participaram com 2,5; 12,8 e $15,3 \%$, respectivamente. Dentre as gramíneas,
Andropogon lateralis, Pennisetum clandestinum e Sporobolus indicus tiveram maior participação na MS da pastagem nativa, com 19, 13 e 11,8\%, respectivamente. Outras gramíneas com participação importante na composição florística foram Paspalum notatum, Paspalum nicorae e Paspalum plicatulum, com participação na MS de 8,6 e $2 \%$. As espécies de compostas com maior destaque foram Elephantopus mollis, Hypochoeris sp., Aspilia montevidensis e Chevreulia sarmentosa, com participação de 8, 1, 0,8 e $0,6 \%$ na MS. Dentre as espécies de hábito cespitoso que predominam na pastagem nativa, destacam-se Andropogon lateralis e Sporobolus indicus. As espécies rizomatosas e/ou estoloníferas foram Pennisetum clandestinum, Paspalum notatum, Paspalum nicorae, Elephantopus mollis, Hypochoeris sp. e Chevreulia sarmentosa. Nesse ambiente de espécies heterogêneas e estrutura vegetal variável (densidade e altura) modificada pelo pastejo seletivo de herbívoros, seguramente, ocorreu uma 
maior variabilidade nas medidas obtidas com disco à medida que aumentou a área do disco.

O efeito espoliativo do pastejo na pastagem nativa constituída de espécies cespitosas e com grande quantidade de colmos rígidos foi responsável pela baixa correlação das leituras obtidas com disco na estimativa de forragem (GOMES et al., 1998). Amenor sensibilidade do disco de avaliação de pasto frente às alterações ocorridas na vegetação nativa sob pastejo, exigindo frequentes calibrações, foi verificada em quatro níveis de $\mathrm{MF}$, com menores coeficientes de determinação $\left(\mathrm{R}^{2}\right)$, para maiores níveis de MF (BARCELLOS, 1990). Conforme o mesmo autor, os resultados da forragem da pastagem nativa apresentaram variações nos valores de $\mathrm{R}^{2}$ entre 73,35 e $36 \%$ e $C V$ entre 37,6 e $54,4 \%$, com um acentuado aumento no $\mathrm{CV}$ nos níveis três e quatro. A imprecisão do disco também foi associada por BANDINELLI et al. (2003) à variação da estrutura da pastagem de Avena strigosa Schreb. e Lolium multiflorum Lam. após o início do pastejo. Segundo os autores, os colmos de Avena strigosa no estrato superior da pastagem, após o pastejo, impediram que o disco medisse a altura real da pastagem.

Medindo a MF de pasto anual de Lolium multiflorum com disco medidor de área $0,1 \mathrm{~m}^{2}$, CAUDURO et al. (2006) observaram baixa correlação entre o aparelho e a MF manejada com alta oferta de forragem no pré e no pós-pastejo, com CVs de 15,98 a $36,21 \%$, mas inferiores aos resultados da presente pesquisa para a mesma área de disco, com acréscimos de pesos. BARCELLOS (1990) referiu-se à melhor adequação do disco medidor de forragem a pastagens puras ou de composição botânica pouco complexa e com espécies de hábito de crescimento semelhantes. Contrariando os resultados obtidos por BARCELLOS (1990) em pastagem nativa submetida a quatro pressões de pastejo, MOOJEN (1991) obteve alta precisão na quantificação da MF com disco medidor de forragem (área $0,25 \mathrm{~m}^{2}$ e peso $9,2 \mathrm{~kg} \mathrm{~m}^{-2}$ ). $\mathrm{O}$ autor verificou valores de $\mathrm{R}^{2}$ superiores a $80 \%$ na média das amostragens para as quatro estações do ano.

Revisando métodos de estimativas de MF, 'T MANNETJE (2000) confirmou que o método de disco foi mais preciso para pastagens rasteiras, como Lolium multiflorum Lam. e Trifolium repens, e que o uso em pastagem cultivada com gramíneas de porte alto, com maior proporção de caule/folha, diminui a precisão da MF. Conforme o autor, medidas de altura de MF e produção de MS devem ser ajustadas para cada tipo de pastagem e mudanças na sua estrutura.

Comparando a estimativa de MF com disco de área de $0,2 \mathrm{~m}^{2}$ e pesos de 5,10 e $15 \mathrm{~kg} \mathrm{~m}^{-2}$ com áreas de 0,$4 ; 0,6$ e $0,8 \mathrm{~m}^{2}$ e peso de $5 \mathrm{~kg} \mathrm{~m}^{-2} \mathrm{em}$ pastagem de
Festuca arundinacea Schreb., BRANSBY et al. (1977) não verificaram maior eficiência entre áreas e pesos de disco para o $\mathrm{CV}$ e o $\mathrm{R}^{2}$. Os autores associaram a insensibilidade dos discos à densidade e à altura da pastagem.

SANTILLAN et al. (1979), em pastagens de Lolium multiflorum Lam., Paspalum notatum e Hemarthria altissima, também não observaram diferenças para diferentes áreas de disco de 0,25; 0,5 e $0,75 \mathrm{~m}^{2}$ e pesos de 1,$14 ; 2,28$ e $3,4 \mathrm{~kg} \mathrm{~m}^{-2}$. No entanto, aumentos de área de disco de 0,25 para $0,75 \mathrm{~m}^{2}$ promoveram valores de correlação (r) maiores e s menores. Áreas de 0,5 e $0,75 \mathrm{~m}^{2}$ não diferiram entre si.

As pesquisas anteriores relacionadas com o emprego de disco medidor não observaram aumentos na eficiência da medida de forragem em pastagens cultivadas com acréscimos de áreas e pesos. Por outro lado, os resultados observados com emprego do disco medidor de forragem de mesma área e peso em pastagem nativa no RS foram contraditórios, resultando em alta e baixa eficiência na estimativa de MF (MOOJEN 1991; BARCELLOS 1990). O resultado verificado na presente pesquisa indicou maior precisão para o disco de menor área e peso avaliado numa vegetação heterogênea e suscetível a modificações estruturais de pastejo em pastagem nativa.

O menor peso de disco de $5 \mathrm{~kg} \mathrm{~m}^{-2}$ associado à menor área apresentou o menor CV (Figura 1b). À medida que pesos foram acrescentados sobre as maiores áreas de disco $\left(0,2\right.$ e $\left.0,3 \mathrm{~m}^{2}\right)$, confirmaram-se elevados valores de CV de 47,6 a $80 \%$ obtidos em pastagem nativa. Os diferentes pesos adicionados sobre a mesma área de disco podem afetar a resistência da pastagem nativa, determinada pela sua estrutura, densidade e altura.

$\mathrm{O}$ menor CV obtido na menor área e peso de disco, provavelmente, englobou vegetação de menor heterogeneidade, em que a pressão máxima pode ter sido atingida com o menor peso. Dessa forma, o acréscimo de pesos resultou no mesmo efeito e, portanto, apenas elevou minimamente o $\mathrm{CV}$ entre as amostragens. Isso também ocorreu na área $0,2 \mathrm{~m}^{2}$, com os acréscimos de pesos no disco. Os baixos coeficientes de determinação $\left(\mathrm{R}^{2}=25\right.$ e $\left.19 \%\right)$ obtidos no modelo de regressão para áreas de disco de 0,1 e $0,2 \mathrm{~m}^{2}$ indicaram que acréscimos de pesos tiveram pouco efeito nas relações das medidas obtidas com disco na estrutura da pastagem nativa. Entretanto, o aumento de pesos na maior área de disco, $0,3 \mathrm{~m}^{2}$, resultou em maior variabilidade nas leituras do disco na vegetação e, portanto, elevou o $\mathrm{CV}$ entre as amostragens $\left(\mathrm{R}^{2}=90 \%\right)$.

Os trabalhos que avaliaram diferentes pesos na mesma área de disco não verificaram efeito do 
acréscimo dos pesos na qualidade das estimativas da MF em pastagens cultivadas, apenas com tendência de diminuir o s (BRANSBY et al., 1977; SANTILLAN et al., 1979). A diminuição do s na estimativa da massa de forragem de pastagens cultivadas com maiores pesos de disco pode estar relacionada às características estruturais da vegetação com composição botânica homogênea e estrutura uniforme. Os maiores CVs verificados nos maiores pesos para mesma área de disco conferiram maiores variações das medidas com disco numa vegetação de pastagem nativa com composição botânica complexa, de espécies de hábitos de crescimento diferentes e modificadas pelo pastejo.

PEDREIRA (2002) relatou diferentes efeitos na estimativa de forragem com diferentes pesos de disco, e discos excessivamente leves responderão apenas à altura, enquanto que discos muito pesados apresentarão pouca relação com a densidade e a altura, promovendo o esmagamento do pasto. Além do menor $\mathrm{CV}$ obtido no disco de área $0,1 \mathrm{~m}^{2}$ e peso $5 \mathrm{~kg} \mathrm{~m}^{-2}$, este apresenta outras vantagens, como facilidade de locomoção e simplicidade no uso.

A relação entre os períodos de avaliação e o $\mathrm{CV}$ foi linear positiva (Figura 1c).

Com o avanço dos períodos estudados, o $\mathrm{CV}$ das medidas obtidas com o disco aumentou linearmente $(\mathrm{P}<0,0001)$, de 32 a $50 \%$, para os períodos determinados entre 07/10/2004 e 16/04/2005. A variabilidade de espécies da vegetação da pastagem nativa e as alterações nos estádios fenológicos das diferentes espécies, com o avanço das estações do ano, influenciaram o CV. Essa diferença seria causada pela maior participação de forragem verde nas épocas de crescimento inicial, com menor peso seco e mais flexibilidade da estrutura da MF. O avanço no estádio de desenvolvimento das plantas forrageiras promovido pelo aumento do teor de MS de 42,35\% (médias das avaliações da primavera) para 56,34\% (médias das avaliações do verão) proporcionou maior resistência da forragem da pastagem nativa.

O comportamento do teor de MS semelhante aos comportamentos observados neste trabalho também foi observado por BARCELLOS (1990), avaliando pastagem nativa sob pastejo com disco medidor de forragem referente às avaliações médias na primavera e no verão. $\mathrm{O}$ autor verificou diferença $(\mathrm{P}<0,01)$ no coeficiente de regressão das equações de 244,6kg de MS na estação da primavera e $351,2 \mathrm{~kg}$ de MS no verão. Os CVs médios das equações de regressão foram de 45,91 e 42,1\% para as referidas épocas. Altas fontes de erro verificadas no mesmo trabalho foram explicadas pelo incremento da forragem seca no final do ciclo fenológico da pastagem nativa e pela demarcada heterogeneidade da vegetação promovida pelo pastejo seletivo, devendo ser acrescentada a menor sensibilidade do disco de avaliação às alterações ocorridas na vegetação. Dificuldades de avaliação e menor consistência na estimativa de forragem com disco medidor de pasto, com área de $0,25 \mathrm{~m}^{2}$ e peso de $9 \mathrm{~kg} \mathrm{~m}^{-2}$, em pastagem nativa consorciada com Lolium multiflorum Lam. e Trifollium vesiculosum Savi., foram relacionadas às variações na condição da pastagem, em termos de MF, pastejo e estado fenológico das espécies no período de inverno e na primavera (BARCELLOS, 1990). Segundo o mesmo autor, outros fatores, como as alterações nos componentes botânicos das espécies avaliadas e modificados pelo pastejo, promoveram a ampla variação dos CVs, de 17 a 45\%, obtidos nas datas de avaliação (21/07 a 10/11).

No Rio Grande do Sul, há necessidade de estudos para determinar o melhor tipo de estimativa de medição de pasto e as condições de pastejo.

\section{CONCLUSÕES}

As estimativas com disco de avaliação em área de pastagem nativa aumentaram o coeficiente de variação residual à medida que a área de disco aumentou e quando pesos foram acrescentados sobre a mesma área de disco. $\mathrm{O}$ teor de matéria seca da pastagem nativa aumentou o coeficiente de variação residual na primavera e no verão.

\section{AGRADECIMENTOS}

Os autores agradecem ao Dr. José Pedro Pereira Trindade, pela colaboração e pelas sugestões na conclusão deste trabalho, bem como o auxílio dos colegas Juliano Roman, Stefani Macari e Adriano Maximer.

\section{REFERÊNCIAS}

BANDINELLI, D.G. et al. Comparação de métodos para estimativa da massa de forragem em gramíneas de estação fria. In: REUNIÃO ANUAL DA SOCIEDADE BRASILEIRA DE ZOOTECNIA, 40., 2003, Santa Maria, RS. Anais... Santa Maria; Sociedade Brasileira de Zootecnia, 2003. CD-ROM.

BARCELLOS, A.O. Avaliação de métodos para estimativas de massa de forragem em condições de pastejo. 1990. 181f. Dissertação (Mestrado em Zootecnia) - Curso de Pósgraduação em Zootecnia, Universidade Federal do Rio Grande do Sul.

BRANSBY, D.I. et al. Disk meter for rapid estimation of herbage yield in grazing trials. Agronomy Journal, Madison, v.69, n.3, p.393-396, 1977.

CAUDURO, G.F. et al. Comparação de métodos de medida indireta de massa de forragem em pasto de azevém anual (Lolium multiflorum 
Lam.). Ciência Rural, v.36, n.5, p.1617-1623, 2006. Disponível em: <http:/www.scielo.br/scielo.php?script=sci_pdf\&pid=S0103$84782006000500044 \& \operatorname{lng}=\mathrm{pt} \& \mathrm{nrm}=\mathrm{iso} \& \mathrm{t} \operatorname{lng}=\mathrm{pt}>$. Acesso em: 22 abr. 2007. doi: 0103.8478/IDSO103-84782006.

FRAME, J. Herbage mass. In: HODGSON, J. et al. Sward measurement hand-book. Berkshire: Hurley, 1981. p.3969.

GOMES, K.E. et al. Efeito de ofertas de forragem, diferimentos e adubações sobre a dinâmica de uma pastagem natural. Acumulação de matéria seca. In: REUNIÃO ANUAL DA SOCIEDADE BRASILEIRA DE ZOOTECNIA, 35., 1998 Botucatu, SP. Anais... Botucatu: Sociedade Brasileira de Zootecnia, 1998. CD-ROM.

IBGE. Censo agropecuário 1995-1996. Online. Disponível em: http:/www.ibge.gov.br/home/estatistica/economia/agropecuaria/ censoagro/1995_1996/default.shtm Acesso em: 02 jun. 2006.

PEDREIRA, C.G.S. Avanços metodológicos na avaliação das pastagens. In: REUNIÃO ANUAL DA SOCIEDADE BRASILEIRA DE ZOOTECNIA, 39., 2002, Recife, PE.
Anais... Recife: Sociedade Brasileira de Zootecnia, 2002. CDROM.

SANTILLAN, R.A. et al. Estimating forage yield with a disk meter. Agronomy Journal, v.71, n.1. p.71- 74, 1979.

SAS INSTITUTE. SAS/STAT. User's guide: statistics, Version 8.02. Cary, 2001. V.1, 890p. V.2, 1686p.

'T MANNETJE, L. Measuring biomass of grassland vegetation. In: 'T MANNETJE, L.; JONES, R.M. Field and laboratory methods for grassland and animal production research. Wallingford: CABI Publishing/CAB International, 2000. p.151177.

TOTHILL, J.C. et al. BOTANAL - A comprehensive sampling and computing procedure for estimating pasture yield and composition. 1. Field sampling. Tropical Agronomy Technical Memorandum, n.78, 24 p, 1992.

WILM, H.G. et al. Estimating forage yield by the doublesampling method. Journal of the American Society of Agronomy, v.36, n.1, p.194-203, 1944. 